Note

\title{
Phenology of the oil palm interspecific hybrid Elaeis oleifera $\times$ Elaeis guineensis
}

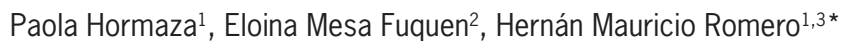

${ }^{1}$ Centro de Investigación en Palma de Aceite/Programa de Biología y Mejoramiento de la Palma de Aceite - Cenipalma, Calle 21 No. 42-55 - 111611 - Bogota - Colombia. ${ }^{2}$ Centro de Investigación en Palma de Aceite/División de Transferencia de Resultados de Investigación. 3Universidad Nacional de Colombia - Depto. de Biología, Carrera 45 No. 26-85 - 111311 - Bogotá - Colombia *Corresponding author <hromero@cenipalma.org>

Edited by: Antonio Costa de Oliveira

Received January 21, 2011

Accepted January 05, 2012
ABSTRACT: Oil palm is one of the most important oil crops in the world. Because of its high productivity and perennial nature, it has been expanding quickly. Commercial plantations consist mostly of the African palm E. guineensis Jacq. However, producers in Latin America are increasingly planting the $O \times G$ interspecific hybrid, a cross between African palm ( $E$. guineensis) and the American palm (E. oleifera (Kunth) Cortés). This interspecific hybrid has emerged as a promising solution to diseases such as the bud rot of oil palm because of the apparent partial resistance of this genotype to the disease. This work studied and described the phenology of the $O \times G$ interspecific hybrid. The phenology stages were coded using the $\mathrm{BBCH}$ scale. The scale for the phenophases was defined using a three-digit code. Due to the nature of the palm, no descriptions were used for stage two (formation of side shoots/tillering) and stage four (development of harvestable vegetative plant parts or vegetative reproductive organs) because these stages do not apply to oil palm. The scale was constructed using germinating seeds, pre-nursery and nursery plants and five year-old palms. For the description of the stem elongation, different age palms of the same $0 \times \mathrm{G}$ hybrid were used. Observations were performed during an 18-month period. Additionally, the interval for the change from one phenology stage to another was determined both in days and degree-days (DD). The interspecific $0 \times \mathrm{G}$ hybrid required $6408 \mathrm{DD}$ from when the spear leaf unfolds until the bunch was ripened and harvested, and 4427.6 DD from leaf unfolding to anthesis.

Keywords: $\mathrm{BBCH}$ scale, degree-days, phenophase

\section{Introduction}

Oil palm is one of the most important crops in the world and by far out-yields other oil crops. There are more than 12 million hectares planted with oil palm in the world, with a production of over 45 million tons, which is expected to increase substantially due to growth in demand for oil by the food industry and the use of Biodiesel in Europe and in some South American countries (Fedepalma, 2010).

Most commercial oil palm plantations in the world consist of African palm E. guineensis Jacq., native to the Gulf of Guinea in West Africa (Ergo, 1997). There is another oil palm species, the American palm Elaeis oleifera, (Kunth) Cortés, which originated in various regions of Central and South America, and which is not commercially exploited. In recent years, due to the presence of diseases, plantations of the $\mathrm{O} \times \mathrm{G}$ interspecific hybrid, a cross between the American palm and the African palm, have increased substantially in Latin America because of its apparent partial resistance to the bud rot disease caused by Phytophthora palmivora (Torres et al., 2010). However, its characteristics of vegetative and reproductive development are unknown, and its phenology has not been described, despite the high potential of the $\mathrm{O}$ $\times \mathrm{G}$ hybrid in the market.

This research was done in order to characterize and chronologically describe the morphological and phenology stages of the interspecific hybrid $(\mathrm{O} \times \mathrm{G})$, according to the extended $\mathrm{BBCH}$ scale and decimal coding (Hack et al., 1992). Also the intervals between phenology stages expressed in days and degree-days were measured.

\section{Materials and Methods}

Experimental data were obtained at Barranca de Upía, Meta, Colombia (40²9' N; 7257' W; 190 m a.s.1.). The average temperature is $26.4{ }^{\circ} \mathrm{C}$, relative humidity of $81 \%$ and annual rainfall of $2589 \mathrm{~mm}$. The study area is located in a warm humid area, with a monomodal rainfall pattern. The period with the highest rainfall occurs from May to Oct., with an average of 20 days. There is a dry period with a lower number of rainy days from Dec. to Mar., averaging one to four days, with an annual average of 150 to 200 rainy days during the evaluation period.

The observations were made during the period from Oct. 2008 to May 2010. During this period there was a cumulative rainfall of $3,372 \mathrm{~mm}$. The periods with highest rain frequency were on Nov. 2008, from May to Jul. 2009 and on May 2010. Average relative humidity was $79 \%$ with a variation between $62 \%$ and $90 \%$. Average temperature was $26.6^{\circ} \mathrm{C}$ with a maximum of 35.7 ${ }^{\circ} \mathrm{C}$ and a minimum of $20.5^{\circ} \mathrm{C}$.

The germination was described by the observation of 190 seeds of interspecific hybrid $\mathrm{O} \times \mathrm{G}$. The description of the initial growth stages (pre-nursery and nursery) was achieved by the observation from seed germination until the time of planting on the field of 24 palms of the interspecific hybrid $\mathrm{O} \times \mathrm{G}$, Coari $\times$ La Mé cross. 
To determine the phenology stages on the adult palms, $20 \mathrm{O} \times \mathrm{G}$ hybrid palm trees, Coari $\times$ La Mé cross, planted on the field in 2005 were used. Within the study plot, selected palms were free of phytosanitary problems and planted in the same type of soil. For the description of the phenology stages of the leaves in the adult plants, leaves that according to their phyllotaxis were in the zero or spear leaf position (leaf with leaflets fully folded against the rachis, and $0 \%$ of its leaflets unfolded) were identified and marked, and observations were made daily until the change of position in the immediately next phyllotaxis (Leaf 1: leaf with $70 \%$ of its leaflets unfolded) and subsequent changes in position of the leaves were time-recorded. Leaf senescence was recorded by the observation of different age leaves of 24 palms of the same cultivar of different ages, up to 13 years old. The same palms were used for the description of stem phenology.

For the reproductive stages, observations were made on 300 reproductive structures produced during the evaluation period by the $20 \mathrm{O} \times \mathrm{G}$ hybrid palm trees, Coari $\times$ La Mé cross, planted on the field in 2005. The observations of these structures started with the emergence of the inflorescence in the axil of each leaf with respect to the axis of the stem. Flowering and changes at this stage, and the development and ripening of fruits and bunches were recorded. To determine fruit and bunch development and ripening stages, the inflorescences in the axils of the leaves that emerged after marking the spear leaf were selected. These inflorescences were hand-pollinated at the time of anthesis with pollen from $E$. guineensis palms, with a germination rate above $80 \%$, using sterile powder as a transport medium for pollen at a 7:1 ratio. Pollinations were performed between 6 h00 and 10h00.

The equatorial growth of the fruit was measured using a digital gauge, from 30 to 105 days after anthesis, and the change in color was also monitored in three fruit sections: basal, middle and apical, up to 162 days after anthesis. Additionally, color variations in the endocarp and variations in shape and texture of the endosperm during the same period of time were described using photographs.

The frequency of observations was set based on the organ observed and on how fast the hybrid phenology changed over time. For example, in the flowering phase, observations of the undifferentiated, pre-anthesis and anthesis stages were made on a daily basis due to the rapid evolution of the visible changes in these organs, while for the development and growth of the stem, data were collected monthly. The $\mathrm{BBCH}$ (Biologische Bundesantalt, Bundessortenamt und Chemische Industrie) General Scale descriptive tool was used for the description of the phenology stages of the plant from seed stage to leaf senescence (Bleiholder et al., 1989; Hack et al., 1992).

The duration of phenology stages for leaf unfolding and inflorescence and fruit development of the dif- ferent materials was measured in days and degree-days. The latter were calculated as the sum of the differences between the average daily temperatures and the base temperature, according to the following equation:

$\mathrm{DD}=(\mathrm{Tmin}-\mathrm{Tb})+(\mathrm{Tmax}-\mathrm{Tmin} / 2) * \mathrm{~N}$

where DD = degree days (heat units), Tmin = minimum air temperature $\left({ }^{\circ} \mathrm{C}\right) ;$ Tmax $=$ maximum air temperature $\left({ }^{\circ} \mathrm{C}\right) ; \mathrm{Tb}=$ base temperature $\left({ }^{\circ} \mathrm{C}\right)$, and $\mathrm{N}=$ the number of days duration of each phenology phase. The equation was used only when $\mathrm{Tb}<\mathrm{Tmin}$.

The temperature baseline for oil palm was taken from a study by Henry (1955), where he noted that at temperatures below $15^{\circ} \mathrm{C}$ the growth of oil palm seedlings is totally inhibited under controlled conditions. For observation of climate variables a WatchDog Weather Station series 2900-ET was used, which records weather data every half hour.

\section{Results and Discussion}

\section{Scale features}

The $\mathrm{BBCH}$ scale for the $\mathrm{O} \times \mathrm{G}$ interspecific hybrid does not use the stage two (formation of side shoots/tillering) and stage four (development of harvestable vegetative plant parts or vegetative reproductive organs / booting) of the general scale. In addition to the use of a two-digit code it was necessary to introduce a third digit between the primary growth stage and secondary growth stage (meso-stage) to allow flexibility in the subdivision of the growth stages. Therefore, the nomenclature of the $\mathrm{BBCH}$ scale for the oil palm interspecific hybrid $(\mathrm{O} \times \mathrm{G})$ contains three digits, where the first digit indicates the primary growth phase (0-9), the second digit correspond to the mesostage (0-9), which is inserted between the primary and secondary stages, and the third digit corresponds to the secondary stages (0-9). The secondary stages are used to accurately describe short phases of plant development.

For the interspecific hybrid $\mathrm{O} \times \mathrm{G}$, vegetative development was described through three primary growth stages: germination and emergence (stage 0), leaf development in greenhouse and field (stage 1), and stem elongation (stage 3). Reproductive growth was associated with four stages: inflorescence emergence (stage 5), flowering (stage 6), fruit growth (stage 7) and fruit ripening (stage 8). Finally, leaf senescence stage was described (stage 9).

\section{Description of the growth stages of the Interspecific Hybrid $(\mathrm{O} \times \mathrm{G})$}

\section{Principal stage 0 - Germination and emergence}

000: Dry seed - Seeds are ovoid to angular and dark brown; the endocarp is covered by a series of cross- 
linked fibers surrounding the apical germinal pores. The endocarp is thin and hard with a thickness between 0.5$2.5 \mathrm{~mm}$.

001: Complete imbibition - The seed can be classified as cryptocotyledonary; initially the cotyledonary petiole emerges, which is whitish and cylindrical, with an elongation of the swollen embryo and its distal end is visible in one of three germination pores, pressing against a disk-shaped bulge called the operculum (Op) until it is detached, to give way to the stage known as "white spot" in which the radicle and plumule will be differentiated (Figure 1).
004: Differentiation of plumule (pl) and radicle (ra) - visible ten to 14 days after germination (Turner and Gillbanks, 2003). They emerge through a cylinder formed by the ligule (Li), (modified leaf that serves as protection). In the upper part of the ligule is the plumule or first plumular sheath that is pointed and light green at the apex; the radicle has a cream color (Figure 1).

005: Formation of lateral roots (rad) - Formation of lateral roots on the primary root. These are the first lateral roots and grow in the upper ring at the joining point of the radicle and the hypocotyl. (Corley and Tinker, 2009).

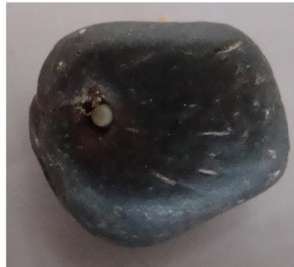

001

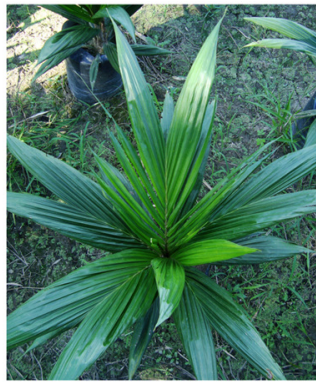

131

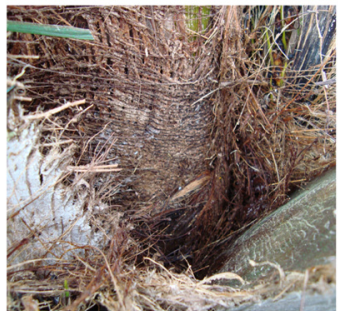

500

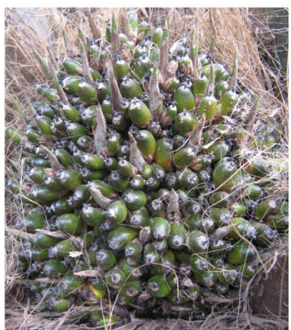

800

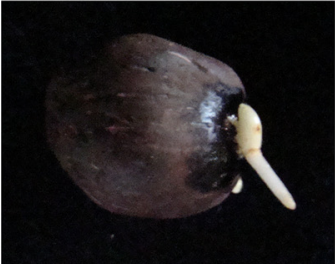

004

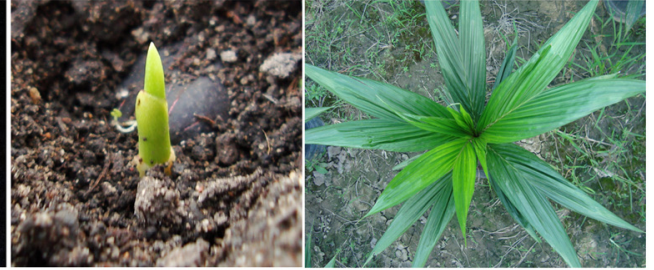

101

129

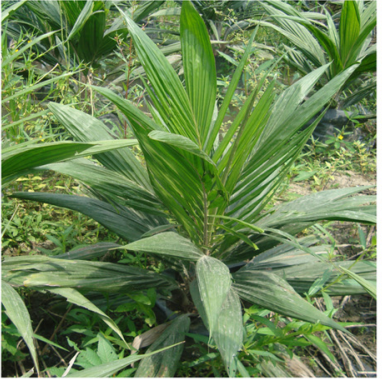

145

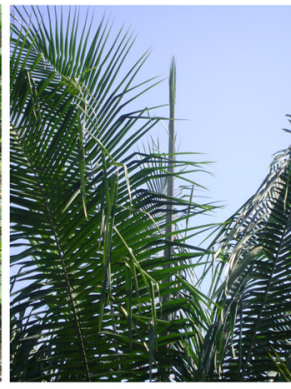

151

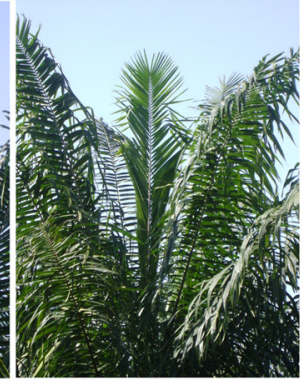

155

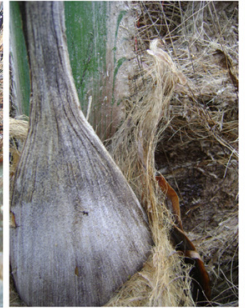

509

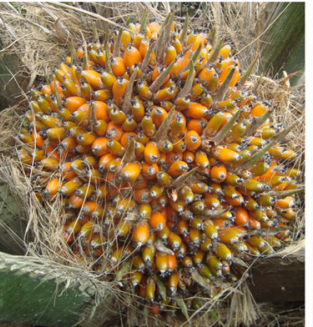

805

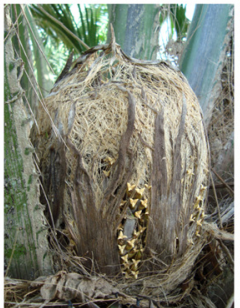

602

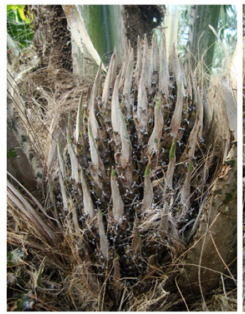

700

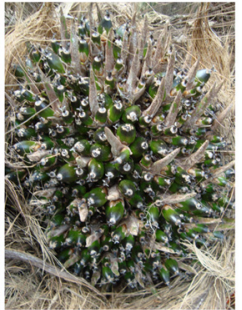

709

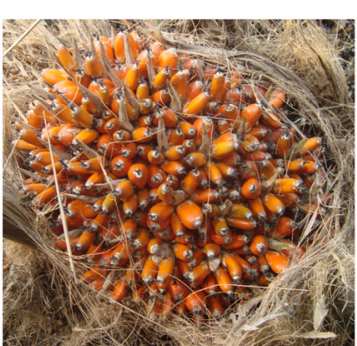

806

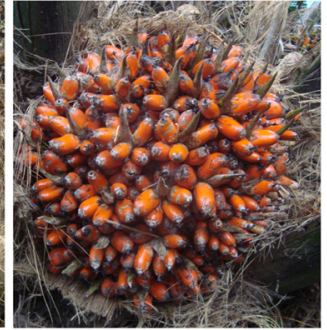

807

Figure 1 - Some phenology stages of the oil palm interspecific hybrid $\mathrm{O} \times \mathrm{G}$ according to the $\mathrm{BBCH}$ (Biologische Bundesantalt, Bundessortenamt und Chemische Industrie) scale. 
009: Two plumular leaves - They emerge from the first plumular sheath and protrude from the ligule until the radicle is one $\mathrm{cm}$ long at which point the plumule emerges through the ligule. The apex of the second plumular leaf breaks through the soil surface. The plumule is light green and the radicle has a cream color.

\section{Principal stage 1 - Leaf development}

\section{Nursery Plants}

101: Emergence of first lanceolate leaf - About a month after emergence, appears a fully expanded leaf blade called "the first lanceolate leaf" (Figure 1).

102: Second lanceolate leaf one month after the emergence of the first leaf.

109: Five or more lanceolate leaves which represent $90 \%$ of development in this stage.

121: First bifurcated leaf - Emerges about five or six months after germination.

129: Five bifurcated leaves - Formation of five leaves bifurcated at the apex (Figure 1).

131: Formation of clefts: Clefts partially divide the area between the veins to form the leaflets, leaf 12 partially pinnate (Figure 1).

141: Two leaf blades - Leaf 18 with clefts that completely divide the veins into two leaf blades, subsequent leaf remains folded. Emergence of pinnate leaves.

143: Leaf 18 fully pinnate and spear leaf $30 \%$ unfolded.

145: Leaf 18 fully pinnate and spear leaf $50 \%$ unfolded (Figure 1).

149: Leaf 18 fully pinnate and spear leaf $70 \%$ unfolded and considered as a new leaf.

\section{Field Plants}

151: $0 \%$ of spear leaf unfolded; rachis fully elongated; leaflets light green tight in the center of the crown. Called leaf 0 or spear leaf. Active upward vertical growth (Figure 1).

153: $30 \%$ of spear leaf unfolded - Leaflets begin to detach from the apex but still folded up; no visible detachment of lower leaflets.

155: $50 \%$ of spear leaf unfolded - $50 \%$ of upper leaflets unfolded; lower part of the leaf still tightly folded towards the center (Figure 1).
159: $70 \%$ of spear leaf unfolded - $70 \%$ of upper leaflets unfolded; lower part of the leaf begins the separation of leaflets from the rachis, but remains folded toward the center, but not tightly. At this stage, this leaf is considered as leaf one (1). After the leaf unfolds its leaflets, it moves progressively outwards and downwards as the younger leaves emerge; the leaflets have now completed their growth in area.

\section{Principal stage 3. Stem elongation}

300: Swelling of the stem base three or four months after germination; the stem has an inverted cone shape; first adventitious roots visible.

301: The stem becomes visible, shaped as an inverted cone. From the nursery stage and up to the first three years, the stem base of the oil palm thickens with no significant growth in height.

305: The stems are solid with strong, broad petiole bases that remain attached to it for about 14 years or more. From there on, leaf bases at the middle of the stem begin to break down and fall off, while leaf bases at the basal and apical sections of the stem remain attached.

309: Stem without petiole bases - There are visible scars from these structures and between them there are marks similar to stem internodes. Some highly decomposed petiole bases remain attached to the stem near the leaf crown.

\section{Principal stage 5: Inflorescence Emergence}

500: Inflorescence not visible; the structure is flattened at the back of the axil firmly attached to the petiole base of the leaf that supports it. This stage is observed between phyllotaxis positions 13 to 15 .

501: Inflorescence visible in the leaf axil due to the onset of the separation of the floral structure from the leaf base that supports it. Emergence of $10 \%$ of final size of the floral structure, elliptical in shape and covered by the floral bract and prophyll fused together. Structure located downward in the opposite direction to the phyllotaxis of the palm; the structure elongates before it thickens. This stage is visible in the phyllotaxis position 15 to 19 .

503: Emergence of $30 \%$ of final size of the floral structure; elongation of the structure.

505: Emergence of $50 \%$ of final size of the floral structure; still closed, and moves one leaf position because of the unfolding of a new leaf. Floral structure begins thickening. The prophyll is torn and disintegrates, while the peduncular bract still covers the structure. 
509: Emergence of $90 \%$ of final size of the floral structure; the prophyll has almost completely disintegrated; a slight tearing can be seen in the central part of the peduncular bract. Inflorescence nearing the start of pre-anthesis stages (Figure 1).

\section{Principal stage 6: Flowering}

601: Pre-anthesis I - Female inflorescence - Beginning of tearing of the peduncular bract in the middle of the structure; dark brown rachillae tight toward the center, no tepals showing.

602: Pre-anthesis II - Female inflorescence - 30 $\%$ tearing of the peduncular bract total area, more open dark brown rachillae; dark brown flower bud tepals showing. Two leaf positions changed since the emergence of the structure.

603: Pre-anthesis III - Female inflorescence $50 \%$ to $70 \%$ tearing of the peduncular bract total area; prophyll disintegrated; light brown separated rachillae; tepals stop growing; burnt-yellow color.

607: Anthesis - Female inflorescence: Fibrous, woody peduncular bract disintegrated and firmly attached to the floral surface; tepals open exposing beige stigma lobes (Figure 1).

609: End of flowering - Beginning of pollination of flowers; progressive change in color of stigma lobes from very dark yellow to brown to black in a 24 hour period; presence of post-pollination exudate on stigmas.

\section{Principal stage 7. Development of fruit}

700: Fertilized female flower: Stigma lobes harden and turn black, beginning of ovary growth; fruit is covered by whorl bracts (Figure 1).

705: $50 \%$ of final fruit size - Beginning of fruit pigmentation to bright light green at the apex; endocarp or endosperm not showing; beginning of rapid growth of fruits (drupes). The fruit is covered by the tepals; no formation of endocarp or endosperm inside the fruit.

707: $65 \%$ of final fruit size - Beginning of fruit pigmentation to dark green at the apex, medium green in the center; no endocarp or endosperm showing. Fruit still covered by whorl bracts. Beginning of formation of endocarp inside the fruit.

708: Fruit reaches normal size for the species - The fruit reaches normal size for the species. The color of the fruit is yellow or very light green in the basal part, light green in the middle and dark green at the apex. Soft endocarp, liquid endosperm. Beginning of solidification of endocarp; endosperm is liquid.
709: Fruit brightness - Fruits are shiny and opalescent green. Endocarp begins hardening and has medium salmon color; endosperm in colloidal state (Figure 1).

\section{Principal stage 8 - Ripening of fruit}

800: Loss of green pigmentation, characteristic of maturity. Between 101 to 105 days after anthesis, fruit color at the base is very light green-yellow, green-yellow in the center and light green at the apex. Endocarp starts hardening, medium brown; white and soft endosperm (Figure 1).

803: Beginning of drastic change in color, halfripened stage - The external coloration of the fruits changes, giving the bunch a tricolor mosaic appearance because fruits are at different stages of development due to the asynchronous anthesis. At this stage, color begins to change from green to orange.

805: Homogenization of bunch coloration - The overall bunch color changes to dark yellow or light orange; fruits are yellow or pale orange at the base, orange in the middle and very light green at the apex. Hard, dark brown endocarp with increased thickness (Figure 1).

806: Beginning of fruit and bunch ripening The overall color of the bunch is medium orange. Fruits are yellow or intense orange at the base, opaque orange in the middle and dark orange at the apex. Fruits not easily detached from the bunch; presence of oil in the mesocarp.

807: Ripening of fruit and bunch - The overall external color of the bunch is intense orange. Fruits are medium orange at the base, intense orange in the middle and dark orange at the apex. Presence of loose fruits. Presence of stretch marks arranged in a circular pattern around the apical part of the fruit. Formation of oil in the mesocarp (Figure 1).

809: Over-ripenning of the fruit and the bunch - Bunches are intense orange, with cracked and more than ten loose fruits.

\section{Principal stage 9: Senescence}

909: Leaf senescence - Old leaves change color from green to yellow and finally turn light brown.

The $\mathrm{BBCH}$ scale for the oil palm interspecific hybrid $\mathrm{O} \times \mathrm{G}$ describes accurately the different developmental stages, including dry seed, leaf development, inflorescence development, flowering, formation and ripening of fruits and bunches. Different agronomic and harvesting tasks for the $\mathrm{O} \times \mathrm{G}$ hybrid are linked to phenology stages based on subjective observations. The $\mathrm{BBCH}$ scale allows phenophases to be standardized so that these tasks can be performed more efficiently and scheduled objectively, according to coded phenology stages. For example, the practice of assisted pollination 
Table 1 - Stages, codes and duration in days and degree-days of the phenology stages spear leaf unfolded, inflorescence emergence, preanthesis I, II, III, anthesis and ripening of fruit and bunch in the interspecific oil palm hybrid $\mathrm{O} \times \mathrm{G}$.

\begin{tabular}{lcccc}
\hline Stage & $\mathrm{BBCH}^{*}$ Code & Duration in days & degree days & Leaf position \\
\hline & & & ${ }^{\circ} \mathrm{C}$ & \\
$70 \%$ of spear leaf unfolded & 159 & $14.4 \pm 0.3$ & $186.9 \pm 4.1$ & $14.7 \pm 0.1$ \\
Inflorescence visible in the leaf axil & 501 & $229.7 \pm 1.0$ & $2843.5 \pm 12.76$ & $20.6 \pm 0.1$ \\
Pre-anthesis I & 601 & $97.7 \pm 1.0$ & $1226.4 \pm 12.0$ & $21.1 \pm 0.1$ \\
Pre-anthesis II & 602 & $4.5 \pm 0.3$ & $72.0 \pm 2.1$ & $21.3 \pm 0.1$ \\
Pre-anthesis III & 603 & $3.3 \pm 0.1$ & $58.1 \pm 1.8$ & $21.4 \pm 0.1$ \\
From PAlll to anthesis & 607 & $2.3 \pm 0.1$ & $40.9 \pm 0.8$ & \\
From antesis to ripening of fruit and bunch & 807 & $168.0 \pm 1.1$ & $1981.1 \pm 14.4$ & \\
Total & & 519.9 & $6,408.7$ & \\
\hline
\end{tabular}

${ }^{*} \mathrm{BBCH}=$ Biologische Bundesantalt, Bundessortenamt und Chemische Industrie.

and the optimal harvesting time can be linked to specific stages as described in this BBCH scale in order to obtain better results in these two tasks. Similarly, researchers in other areas such as entomology and plant pathology could use the $\mathrm{BBCH}$ scale, in order to make more precise correlations, for example, between phenology stages in which the hybrid is more susceptible to the attack by pests or diseases.

One of the items of the scale is the duration (in days) of the stages of leaf development (stage 1), inflorescence emergence (stage five) and flowering (stage six) (Table 1). The number of days of the stages is expected to change according to climate conditions of the study area. Therefore, the duration of each stage is expressed in degree days (DD), taking into account that there is a close relationship between phenophases and degree days, especially in the flowering and leaf development stages (Schwartz, 2003). Thus, estimating the duration of phenophases in terms of degree-days provides a phenological monitoring tool independent of climate variations specific to each area in which the $\mathrm{O} \times \mathrm{G}$ hybrid material is planted.

Under the conditions of the Eastern Zone (Barranca de Upía, Meta), the interspecific hybrid $\mathrm{O} \times \mathrm{G}$ requires 6408 DD and 519.8 days (17.3 months) from when the spear leaf unfolds until the bunch is ripened and harvested (Table 1). It has higher requirements of degree-days from leaf unfolding to anthesis, with 4427.6 DD and 351 days. This may be due to the marked difference between the average daily maximum temperature of $35.7^{\circ} \mathrm{C}$ and average daily minimum of $20.5{ }^{\circ} \mathrm{C}$, which affects the development of the material under these conditions.

It took more than 229 days and 2843.5 DD for the emergence of the undifferentiated inflorescence. Foliar position for this study was in leaf 14 to 15 . It took 98 days and 1226.4 DD for the pre-anthesis I stage to appear, while for the emergence of pre-anthesis stages II and III the number of days and degree days fell sharply to 4.5 days and $71.9 \mathrm{DD}$ and three days and 58.1 DD, respectively. Finally, it took two days and 40.9 DD for the onset of anthesis, with respect to the previous stage of development.

\section{Acknowledgements}

To the Oil Palm Development Fund managed by Fedepalma, and the Administrative Department of Science, Technology and Innovation - Colciencias (contract 426-2007) for funding this research project. Special thanks also to the Guaicaramo S.A Plantation for its support and provision of logistics for phenological monitoring.

\section{References}

Bleiholder, H.; van den Boom, T.; Langelu"ddeke, P.; Stauss, R. 1989. Standardized coding of phenological stages of crops and weeds. Gesunde Pflanzen 41: 381-384 (in German).

Corley, R.V.H.; Tinker, P.B. 2003. The Oil Palm. 4.ed. Blackwell Science,

Oxford, UK.

Ergo, A.B. 1997. New evidence for the African origin of Elaeis guineensis Jacq. by the discovery of fossil seeds in Uganda. Annales de Gembloux 102: 191-201 (in French, with abstract in English).

Federación Nacional de Cultivadores de Palma de Aceite [Fedepalma]. 2010. Statistical Yearbook 2010. Fedepalma, Bogotá, Colombia.

Hack, H.; Bleiholder, H.; Buhr, L.; Meier, U.; Schnock-Fricke, E.; Weber, E.; Witzenberger, A. 1992. Standardized coding of the phenological development stages of mono and dicotyledonous plants; the general extended $\mathrm{BBCH}$ scale. Nachrichtenbl Deutsch Pflanzenschutzd 44: 265-270 (in German, with abstract in English).

Henry, P. 1955. Leaf growth morphology in Elaeis. Revue Générale de Botanique 32: 66-77 (in French).

Schwartz, M.D. 2003. Phenoclimatic Measures. p. 331-334 In: Schwartz, M.D., ed. Phenology: an integrative environmental science. Kluwer Academic Press, Dordrecht, Netherlands.

Torres, G.A.; Sarria, G.A.; Varon, F.; Coffey, M.D.; Elliot, M.; Martinez, G. 2010. First report of bud rot caused by Phytophthora palmivora on African oil palm in Colombia. Plant Disease 94: 1163.

Turner, P.D.; Gillbanks, R.A. 2003. Oil palm cultivation and management. Incorporated Society of Planters, Kuala Lumpur, Malaysia. 